\title{
Symmetric Bipartite Graphs and Graphs with Loops
}

\author{
Grant Cairns $\|^{*} \quad$ Stacey Mendan $\|^{\dagger}$
}

Department of Mathematics and Statistics, La Trobe University, Melbourne, Australia

received $3^{\text {rd }}$ June 2014, revised $29^{\text {th }}$ Jan. 2015, accepted $3^{\text {rd }}$ Feb. 2015.

We show that if the two parts of a finite bipartite graph have the same degree sequence, then there is a bipartite graph, with the same degree sequences, which is symmetric, in that it has an involutive graph automorphism that interchanges its two parts. To prove this, we study the relationship between symmetric bipartite graphs and graphs with loops.

Keywords: bipartite graphs, degree sequence, symmetry

\section{Introduction}

We say that a finite sequence $\underline{d}$ of nonnegative integers is bipartite graphic if $\underline{d}$ can be realized as the degree sequence of both parts of a bipartite simple graph. For example, Figure 1 gives two realizations of the sequence $(2,2,1,1)$. Notice that the realization on the left is symmetric, while the one on the right is not, where by symmetric we use the following natural definition.
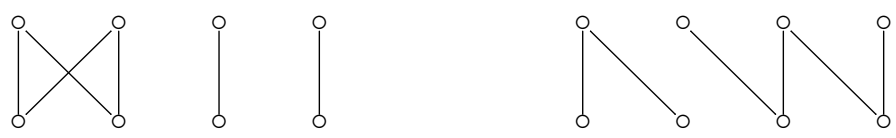

Fig. 1:

Definition 1 We say that a bipartite graph $G$ is symmetric if there is an involutive graph automorphism of $G$ that interchanges its two parts.

We will establish the following result.

Theorem 1 If a sequence $\underline{d}$ is bipartite graphic, then there is a realization of $\underline{d}$ that is symmetric.

We are not aware of any similar result in the literature. Our proof of Theorem 1 relies on an observation connecting symmetric bipartite graphs with graphs-with-loops.

Definition 2 By a graph-with-loops we mean a graph, without multiple edges, in which there is at most one loop at each vertex.

*Email: G.Cairnselatrobe.edu.au.

†Email: spmendan@students. latrobe.edu.au.

1365-8050 (C) 2015 Discrete Mathematics and Theoretical Computer Science (DMTCS), Nancy, France 

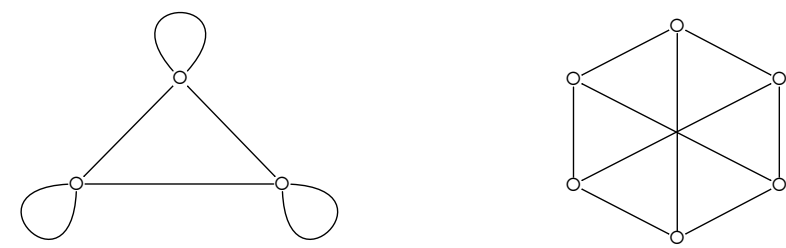

Fig. 2: The complete graph-with-loops on three vertices, and its bipartite double-cover.

Given a symmetric bipartite graph $G$ with involution $\sigma$, the quotient graph $G / \sigma$ has as its vertex set the set of (unordered) pairs $\bar{v}:=\{v, \sigma(v)\}$, for vertices $v$ of $G$, and there is an edge in $G / \sigma$ between $\bar{v}$ and $\bar{w}$ if there is an edge in $G$ between $v$ and $w$ or between $v$ and $\sigma(w)$. So the quotient graph $G / \sigma$ is a graph-with-loops. To see how this process can be reversed, recall that for every simple graph $G$, there is a natural associated bipartite simple graph $\hat{G}$ called the bipartite double-cover of $G$. The graph $\hat{G}$ is the tensor product $G \times K_{2}$ of $G$ with the connected graph $K_{2}$ with 2 vertices; the vertex set of $G \times K_{2}$ is the Cartesian product of the vertices of $G$ and $K_{2}$, there are edges in $G \times K_{2}$ between $(a, 0)$ and $(b, 1)$ and between $(a, 1)$ and $(b, 0)$ if and only if there is an edge in $G$ between $a$ and $b$; see [HIK11]. By construction, $\hat{G}$ is bipartite and symmetric; the automorphism is the map $\sigma:(a, x) \mapsto(a, 1-x)$, and $G$ is the quotient graph $\hat{G} / \sigma$. When $G$ is a graph-with-loops, the above construction again produces a symmetric bipartite graph; each loop in $G$ produces just one edge in $G \times K_{2}$. Figure 2 shows the construction for the complete graph-with-loops $G$ on three vertices.

Note that graphs-with-loops are a special family of multigraphs [Die10] and that for multigraphs, the degree of a vertex is usually taken to be the number of edges incident to the vertex, with loops counted twice. For our purposes, a different definition of degree is more appropriate. We introduce the following definition.

Definition 3 For a graph-with-loops, the reduced degree of a vertex is taken to be the number of edges incident to the vertex, with loops counted once.

So, for example, in the complete graph-with-loops $G$ on three vertices, shown on the left in Figure 2 the vertices each have reduced degree three. The vertices in the tensor product $\hat{G}=G \times K_{2}$ also have the same degrees as the reduced degrees of the corresponding vertices of $G$. In general, if $\underline{d}$ is the sequence of reduced degrees of the vertices of a graph-with-loops $G$, then $\hat{G}$ is a symmetric bipartite graph whose parts have degree sequences $\underline{d}$.

We will employ the following Erdôs-Gallai type result; the proof is given in the final section.

Theorem 2 Let $\underline{d}=\left(d_{1}, \ldots, d_{n}\right)$ be a sequence of nonnegative integers in decreasing order. Then $\underline{d}$ is the sequence of reduced degrees of the vertices of a graph-with-loops if and only iffor each integer $k$ with $1 \leq k \leq n$,

$$
\sum_{i=1}^{k} d_{i} \leq k^{2}+\sum_{i=k+1}^{n} \min \left\{k, d_{i}\right\} .
$$

Note that here, and elsewhere in this paper, the term "decreasing" is taken in the non-strict sense; in other words, non-increasing. Theorem 2 has the following application. 
Theorem 3 A sequence $\underline{d}=\left(d_{1}, \ldots, d_{n}\right)$ of nonnegative integers in decreasing order is the sequence of reduced degrees of the vertices of a graph-with-loops if and only if $\underline{d}$ is bipartite graphic.

Proof: If $\underline{d}$ is the sequence of reduced degrees of the vertices of a graph-with-loops $G$, then the bipartite double-cover $\hat{G}$ of $G$ has parts with degree sequences $\underline{d}$. Conversely, if $\underline{d}$ is bipartite graphic, then by the Gale-Ryser Theorem [Gal57, Rys57], for each $k$ with $1 \leq k \leq n$,

$$
\sum_{i=1}^{k} d_{i} \leq \sum_{i=1}^{n} \min \left\{k, d_{i}\right\} \leq k^{2}+\sum_{i=k+1}^{n} \min \left\{k, d_{i}\right\},
$$

and so by Theorem $2 \underline{d}$ is the sequence of reduced degrees of the vertices of a graph-with-loops.

We can now prove our main result.

Proof of Theorem 1 If If $\underline{d}$ is bipartite graphic, then by Theorem $3 \underline{d}$ is the sequence of reduced degrees of the vertices of a graph-with-loops $G$. Then the bipartite double-cover $\hat{G}$ of $G$ is symmetric and its parts have degree sequences $\underline{d}$.

\section{Some Remarks}

Remark 1 From Theorem 2 and Theorem 3 condition (1) gives an Erdôs-Gallai type condition for a sequence to be bipartite graphic, which is analogous to the Gale-Ryser condition.

Remark 2 It is clear from the discussion in Section 1 that if a sequence $\left(d_{1}, \ldots, d_{n}\right)$ is graphic, then by adding a loop at each vertex, $\left(d_{1}+1, d_{2}+1, \ldots, d_{n}+1\right)$ is the sequence of reduced degrees of the vertices of a graph-with-loops, and so by Theorem 3 the sequence $\left(d_{1}+1, d_{2}+1, \ldots, d_{n}+1\right)$ is bipartite graphic. Note that the converse is not true; for example, $(4,4,2,2)$ is bipartite graphic, while $(3,3,1,1)$ is not graphic.

Remark 3 There are several results in the literature of the following kind: if $\underline{\mathrm{d}}$ is graphic, and if $\underline{\mathrm{d}}^{\prime}$ is obtained from $\underline{\mathrm{d}}$ using a particular construction, then $\underline{\mathrm{d}}^{\prime}$ is also graphic. The Kleitman-Wang Theorem is of this kind [KW73]. Another useful result is implicit in Choudum's proof [Cho86] of the ErdósGallai Theorem: If a decreasing sequence $\underline{\mathrm{d}}=\left(d_{1}, \ldots, d_{n}\right)$ of positive integers is graphic, then so is the sequence $\underline{\mathrm{d}}^{\prime}$ obtained by reducing both $d_{1}$ and $d_{n}$ by one. Analogously, our proof of Theorem 2 . which is modelled on Choudum's proof, also establishes the following result: If a decreasing sequence $\underline{\mathrm{d}}=\left(d_{1}, \ldots, d_{n}\right)$ of positive integers is bipartite graphic, then so is the sequence $\underline{\mathrm{d}}^{\prime}$ obtained by reducing both $d_{1}$ and $d_{n}$ by one.

Remark 4 The authors have pursued related ideas in [CM. CMN CMN14]. In particular, an application of Theorem 3 is given in [CMN14]. For criteria for sequences to be realized by multigraphs, see [MV09]. For further background on graphic sequences, see [BHJW12] TT08, TV03, Yin09] YL05].

\section{Proof of Theorem 2}

The following proof mimics Choudum's proof of the Erdős-Gallai Theorem [Cho86].

For the proof of necessity, consider the set $S$ comprised of the first $k$ vertices. The left hand side of (1) is the number of half-edges incident to $S$, with each loop counting as one. On the right hand side, $k^{2}$ is the 
number of half-edges in the complete graph-with-loops on $S$, again with each loop counting as one, while $\sum_{i=k+1}^{n} \min \left\{k, d_{i}\right\}$ is the maximum number of edges that could join vertices in $S$ to vertices outside $S$. So (1) is obvious.

Conversely, suppose that $\underline{d}=\left(d_{1}, \ldots, d_{n}\right)$ verifies (1) and consider the sequence $\underline{d}^{\prime}$ obtained by reducing both $d_{1}$ and $d_{n}$ by 1 . Let $\underline{d}^{\prime \prime}$ denote the sequence obtained by reordering $\underline{d}^{\prime}$ so as to be decreasing. Suppose that $\underline{d}^{\prime \prime}$ satisfies (1) and hence by the inductive hypothesis, there is a graph-with-loops $G^{\prime}$ that realizes $\underline{d}^{\prime}$. We will show how $\underline{d}$ can be realized. Let the vertices of $G^{\prime}$ be labelled $v_{1}, \ldots, v_{n}$. If there is no edge in $G^{\prime}$ connecting $v_{1}$ to $v_{n}$, then add one; this gives a graph-with-loops $G$ that realizes $\underline{d}$. Similarly, if there is neither a loop at $v_{1}$ nor at $v_{n}$, just add loops at both $v_{1}$ and $v_{n}$. So it remains to treat the case where there is an edge in $G^{\prime}$ connecting $v_{1}$ to $v_{n}$, and at least one of the vertices $v_{1}, v_{n}$ has a loop.

Now, for the moment, let us assume there is a loop in $G^{\prime}$ at $v_{1}$. Applying the hypothesis to $\underline{d}$, using $k=1$ gives

$$
d_{1} \leq 1+\sum_{i=2}^{n} \min \left\{1, d_{i}\right\} \leq n,
$$

and so $d_{1}-2<n-1$. Now in $G^{\prime}$, the degree of $v_{1}$ is $d_{1}-1$ and so apart from the loop at $v_{1}$, there are a further $d_{1}-2$ edges incident to $v_{1}$. So in $G^{\prime}$, there is some vertex $v_{i} \neq v_{1}$, for which there is no edge from $v_{1}$ to $v_{i}$. Note that $d_{i}^{\prime}>d_{n}^{\prime}$. If there is a loop in $G^{\prime}$ at $v_{n}$, or if there is no loop at $v_{i}$ nor at $v_{n}$, then there is a vertex $v_{j}$ such that there is an edge in $G^{\prime}$ from $v_{i}$ to $v_{j}$, but there is no edge from $v_{j}$ to $v_{n}$. Now remove the edge $v_{i} v_{j}$, and put in edges from $v_{1}$ to $v_{i}$, and from $v_{j}$ to $v_{n}$, as in Figure 3 This gives a graph-with-loops $G$ that realizes $\underline{d}$. If there is no loop in $G^{\prime}$ at $v_{n}$, but there is a loop at $v_{i}$, remove the loop at $v_{i}$, add the edge $v_{1} v_{i}$ and add a loop at $v_{n}$, as in Figure 4
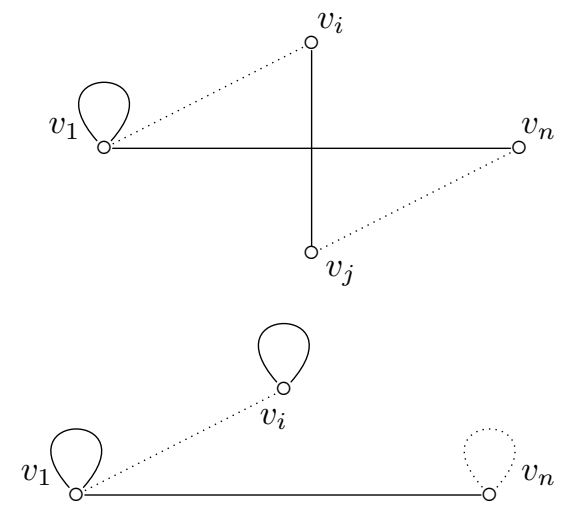

Fig. 3:
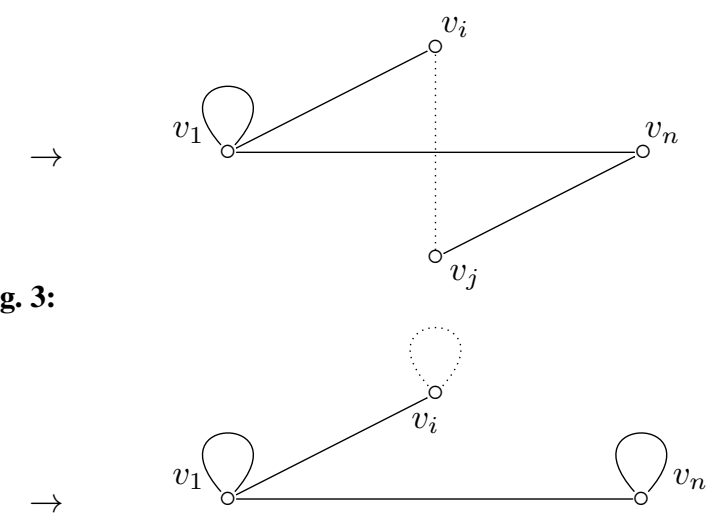

Fig. 4:

Finally, assume there is no loop in $G^{\prime}$ at $v_{1}$, but there is a loop in $G^{\prime}$ at $v_{n}$. So, apart from the loop, there are a further $d_{n}-2$ edges incident to $v_{n}$. Since $d_{1} \geq d_{n}$, we have $d_{1}-1>d_{n}-2$, and so there is a vertex $v_{i}$ such that there is an edge in $G^{\prime}$ from $v_{1}$ to $v_{i}$, but there is no edge from $v_{i}$ to $v_{n}$. Note that $d_{i}^{\prime}>d_{n}^{\prime}$, so as there is a loop in $G^{\prime}$ at $v_{n}$, there is a vertex $v_{j}$ such that there is an edge in $G^{\prime}$ from $v_{i}$ to $v_{j}$, but there is no edge from $v_{j}$ to $v_{n}$. Now remove the loop at $v_{n}$ and the edge $v_{i} v_{j}$, and put edges $v_{j} v_{n}$ and $v_{i} v_{n}$ and add a loop at $v_{1}$, as in Figure 5 This gives a graph-with-loops $G$ that realizes $\underline{d}$. 


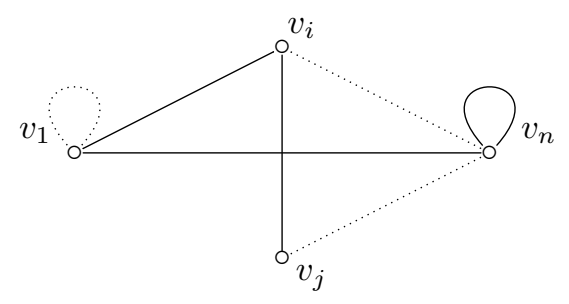

Fig. 5:

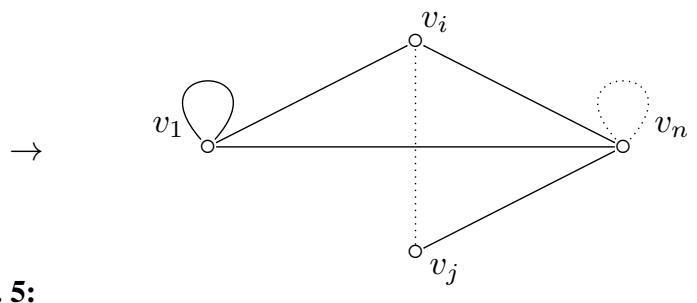

It remains to show that $\underline{d}^{\prime \prime}$ satisfies (1). Define $m$ as follows: if the $d_{i}$ are all equal, put $m=n-1$, otherwise, define $m$ by the condition that $d_{1}=\cdots=d_{m}$ and $d_{m}>d_{m+1}$. We have $d_{i}^{\prime \prime}=d_{i}$ for all $i \neq m, n$, while $d_{m}^{\prime \prime}=d_{m}-1$ and $d_{n}^{\prime \prime}=d_{n}-1$. Consider condition (1) for $\underline{d}^{\prime \prime}$ :

$$
\sum_{i=1}^{k} d_{i}^{\prime \prime} \leq k^{2}+\sum_{i=k+1}^{n} \min \left\{k, d_{i}^{\prime \prime}\right\}
$$

For $m \leq k<n$, we have $\sum_{i=1}^{k} d_{i}^{\prime \prime}=\sum_{i=1}^{k} d_{i}-1$, while $\sum_{i=k+1}^{n} \min \left\{k, d_{i}^{\prime \prime}\right\} \geq \sum_{i=k+1}^{n} \min \left\{k, d_{i}\right\}-1$, and so (2) holds. For $k=n, \sum_{i=1}^{k} d_{i}^{\prime \prime}=\sum_{i=1}^{k} d_{i}-2<k^{2}$, and so (2) again holds. For $k<m$, first note that if $d_{k} \leq k$, then $\sum_{i=1}^{k} d_{i}^{\prime \prime}=\sum_{i=1}^{k} d_{i} \leq k^{2} \leq k^{2}+\sum_{i=k+1}^{n} \min \left\{k, d_{i}^{\prime \prime}\right\}$. So it remains to deal with the case where $k<m$ and $d_{k}>k$. We have

$$
\sum_{i=1}^{k} d_{i}^{\prime \prime}=\sum_{i=1}^{k} d_{i} \leq k^{2}+\sum_{i=k+1}^{n} \min \left\{k, d_{i}\right\}
$$

Notice that as $d_{i}=d_{i}^{\prime \prime}$ except for $i=m, n$, we have $\min \left\{k, d_{i}^{\prime \prime}\right\}=\min \left\{k, d_{i}\right\}$ except possibly for $i=m, n$. In fact, as $k<m$, we have $d_{m}=d_{k}>k$ and $d_{m}^{\prime \prime}=d_{m}-1 \geq k$ and so $\min \left\{k, d_{m}\right\}=$ $k=\min \left\{k, d_{m}^{\prime \prime}\right\}$. Hence $\sum_{i=k+1}^{n} \min \left\{k, d_{i}^{\prime \prime}\right\} \geq \sum_{i=k+1}^{n} \min \left\{k, d_{i}\right\}-1$. Thus, in order to establish 22), it suffices to show that $\sum_{i=1}^{k} d_{i}<k^{2}+\sum_{i=k+1}^{n} \min \left\{k, d_{i}\right\}$. Suppose instead that $\sum_{i=1}^{k} d_{i} \geq$ $k^{2}+\sum_{i=k+1}^{n} \min \left\{k, d_{i}\right\}$. We have

$$
k d_{m}=\sum_{i=1}^{k} d_{i} \geq k^{2}+\sum_{i=k+1}^{n} \min \left\{k, d_{i}\right\}
$$

and so

$$
d_{m} \geq k+\frac{1}{k} \sum_{i=k+1}^{n} \min \left\{k, d_{i}\right\}
$$

Then

$$
\sum_{i=1}^{k+1} d_{i}=(k+1) d_{m} \geq k(k+1)+\frac{k+1}{k} \sum_{i=k+1}^{n} \min \left\{k, d_{i}\right\} .
$$

We have $d_{k+1}=d_{m}>k$ and so $\min \left\{k, d_{k+1}\right\}=k$. Note that $\sum_{i=k+2}^{n} \min \left\{k, d_{i}\right\} \neq 0$ as $k+2 \leq n$, since $k<m \leq n-1$. So 


$$
\sum_{i=1}^{k+1} d_{i} \geq k(k+1)+(k+1)+\frac{k+1}{k} \sum_{i=k+2}^{n} \min \left\{k, d_{i}\right\}>(k+1)^{2}+\sum_{i=k+2}^{n} \min \left\{k, d_{i}\right\},
$$

contradicting (1). Hence $\underline{d}^{\prime \prime}$ satisfies (2), as claimed. This completes the proof.

\section{Acknowledgements}

This study began in 2010 during evening seminars on the mathematics of the internet conducted by the $Q$ Society. The first author would like to thank the Q-Society members for their involvement, and particularly Marcel Jackson for his thought provoking questions. We also thank Yuri Nikolayevsky, whose comments improved the presentation of the paper.

\section{References}

[BHJW12] Michael D. Barrus, Stephen G. Hartke, Kyle F. Jao, and Douglas B. West. Length thresholds for graphic lists given fixed largest and smallest entries and bounded gaps. Discrete Math., 312(9):1494-1501, 2012.

[Cho86] S. A. Choudum. A simple proof of the Erdős-Gallai theorem on graph sequences. Bull. Austral. Math. Soc., 33(1):67-70, 1986.

[CM] Grant Cairns and Stacey Mendan. An improvement of a result of Zverovich-Zverovich. Ars Math. Contemp., to appear.

[CMN] Grant Cairns, Stacey Mendan, and Yuri Nikolayevsky. A sharp refinement of a result of ZverovichZverovich. Discrete Math., to appear.

[CMN14] Grant Cairns, Stacey Mendan, and Yuri Nikolayevsky. A sharp refinement of a result of Alon, BenShimon and Krivelevich on bipartite graph vertex sequences. Australas. J. Combin., 60(2):217-226, 2014.

[Die10] Reinhard Diestel. Graph theory, volume 173 of Graduate Texts in Mathematics. Springer, Heidelberg, fourth edition, 2010.

[Gal57] David Gale. A theorem on flows in networks. Pacific J. Math., 7:1073-1082, 1957.

[HIK11] Richard Hammack, Wilfried Imrich, and Sandi Klavžar. Handbook of product graphs. Discrete Mathematics and its Applications (Boca Raton). CRC Press, Boca Raton, FL, second edition, 2011. With a foreword by Peter Winkler.

[KW73] D. J. Kleitman and D. L. Wang. Algorithms for constructing graphs and digraphs with given valences and factors. Discrete Math., 6:79-88, 1973.

[MV09] Dirk Meierling and Lutz Volkmann. A remark on degree sequences of multigraphs. Math. Methods Oper. Res., 69(2):369-374, 2009.

[Rys57] H. J. Ryser. Combinatorial properties of matrices of zeros and ones. Canad. J. Math., 9:371-377, 1957.

[TT08] Amitabha Tripathi and Himanshu Tyagi. A simple criterion on degree sequences of graphs. Discrete Appl. Math., 156(18):3513-3517, 2008.

[TV03] Amitabha Tripathi and Sujith Vijay. A note on a theorem of Erdős \& Gallai. Discrete Math., 265(13):417-420, 2003.

[Yin09] Jian-Hua Yin. Conditions for $r$-graphic sequences to be potentially $K_{m+1}^{(r)}$-graphic. Discrete Math., 309(21):6271-6276, 2009.

[YL05] Jian-Hua Yin and Jiong-Sheng Li. Two sufficient conditions for a graphic sequence to have a realization with prescribed clique size. Discrete Math., 301(2-3):218-227, 2005. 